\title{
Primary Hyperparathyroidism Superimposed on a Pre-existing Familial Hypocalciuric Hypercalcemia Diagnosis
}

\author{
Alamin Alkundi and Rabiu Momoh
}

\section{ABSTRACT}

Familial hypocalciuric hypercalcemia is a rare clinical condition of persistently elevated serum calcium and reduced urinary calcium levels with an autosomal dominance inheritance pattern to the three out of four large types of this condition known. This rare condition goes largely undiagnosed as patients are largely asymptomatic and where symptoms are present, other causes of hypercalcemia are considered first. Hyperparathyroidism, super-imposing on FHH, is an even rarer occurrence. We present the case of an adult male with an initial provisional assessment of $\mathrm{FHH}$, which was later confirmed with a genetic study. He went on to develop hyperparathyroidism (with evident enlarged parathyroid glands on Sestamibi parathyroid scan done, and an eventual histologic diagnosis of parathyroid adenoma after surgery). It remains to be established if this is an incidental occurrence or if there is a causal relationship between FHH and an onward development of parathyroid hypertrophy or adenoma(ta).

Keywords: Familial hypocalciuric hypercalcemia, genetics, hyperparathyroidism, parathyroid adenoma(ta).

Submitted : August 22, 2021

Published : September 14, 2021

ISSN: 2593-8339

DOI: $10.24018 /$ ejmed.2021.3.5.1023

\section{Alamin Alkundi}

Consultant Endocrinologist, Department of Endocrinology, William Harvey Hospital, Ashford, Kent, TN24 0LZ, United Kingdom.

(e-mail: alamin.alkundi@nhs.net) Rabiu Momoh*

Department of Intensive Care Medicine, William Harvey Hospital, Ashford, Kent, TN24 0LZ, United Kingdom.

(e-mail: rabiu.momoh@nhs.net)

*Corresponding Author

\section{INTRODUCTION}

In the follow-up care given to Familial Hypocalciuric Hypercalcemia (FHH) patients, a possible onward development of hyperparathyroidism should be borne in mind [1]. We report the case of a 52-year-old gentleman who was evaluated a decade prior for symptomatic hypercalcemia and a clinical suspicion for FHH was held out based on history, biochemical and urine studies. While being investigated for a globus sensation, he was found with elevated parathyroid hormone levels and a Sestamibi parathyroid scan study revealed enlarged parathyroid glands on the background of a provisional diagnosis of FHH. Ultrasound study of his thyroid gland also revealed a nodule within the right thyroid lobe. Genetic sequencing study was eventually done to confirm the FHH diagnosis ahead of a slated subtotal thyroidectomy and subtotal parathyroidectomy. Histological studies revealed a follicular adenoma in the thyroid specimen and adenoma on the parathyroid specimen.

\section{CASE}

A gentleman, aged 52, was evaluated ten years prior for intermittent generalized bony aches and recurrent headaches. He had no significant co-morbidities on records at the time and used occasional paracetamol or ibuprofen. He was seen in orthopedic and neurology clinics at that time for these complaints. While being evaluated, persistent hypercalcemia was noted (Table I) with parathyroid hormone level within normal limits. He was symptomatically managed and referred to an endocrinology clinic.

TABLE I: SHOWING CHRONIC HYPERCALCEMIA

\begin{tabular}{|c|c|c|c|c|c|}
\hline $\begin{array}{c}\text { Dates } \\
\text { (dd-mm-yy) }\end{array}$ & $\begin{array}{c}\text { Corrected Serum ionized calcium } \\
\text { levels (ref. } 2.2-2.6 \mathrm{mmol} / 1)\end{array}$ & Dates & $\begin{array}{l}\text { Corrected Serum calcium } \\
\text { levels (ref. } 2.2-2.6 \mathrm{mmol} / \mathrm{l})\end{array}$ & Dates & $\begin{array}{l}\text { Corrected Serum calcium } \\
\text { levels (ref. } 2.2-2.6 \mathrm{mmol} / \mathrm{l}) \\
\end{array}$ \\
\hline 19.6 .20 & 2.8 & 4.3 .19 & 2.9 & 5.8 .14 & 2.8 \\
\hline 12.2 .20 & 2.7 & 27.2.19 & 2.9 & 8.1 .14 & 2.8 \\
\hline 16.11 .19 & 2.6 & 24.1 .19 & 3.0 & 17.7.13 & 2.9 \\
\hline 12.10 .19 & 2.8 & 27.12 .18 & 3.0 & 22.5 .2013 & 2.8 \\
\hline 20.8 .19 & 2.8 & 18.12 .18 & 3.0 & 25.2 .2012 & 2.7 \\
\hline 23.7 .19 & 2.6 & 3.9 .18 & 2.9 & 19.5.11 & 2.8 \\
\hline 1.7 .19 & 2.8 & 9.8 .17 & 2.9 & & \\
\hline 24.6 .19 & 2.9 & 28.12 .16 & 2.8 & & \\
\hline 9.5 .2019 & 2.9 & 31.1 .15 & 2.9 & & \\
\hline
\end{tabular}


Upon evaluation at the endocrinology clinic, he was noted to have had a normal parathyroid uptake scan at the time. Based on laboratory evidence of persistent hypercalcemia and hypocalciuria (Fig. 1), low calcium : creatinine ratio, and a report of asymptomatic hypercalcemia in patient's son, a provisional diagnosis of FHH was held. Increased fluid intake and oral acetaminophen were recommended to alleviate his symptoms. Follow-up blood workups and screening for renal hypercalcemia complications (stones and worsening renal function) were planned.

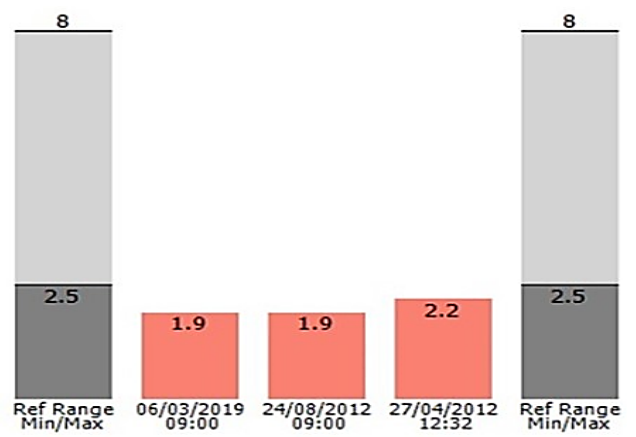

Fig. 1. Showing chronic hypocalciuria.

After a five-year hiatus, he was seen again at the endocrinology clinic with corrected serum ionized calcium level at $2.9 \mathrm{mmol} / \mathrm{l}$ and with a normal renal function study (serum creatinine $77 \mathrm{umol} / \mathrm{l}$ and EGFR of $101 \mathrm{ml} / \mathrm{min}$ ). Bone scan done showed no osteoporosis, and renal ultrasound scan done showed no renal stones. On examination, his blood pressure was $126 / 76 \mathrm{mmHg}$, weight $86.8 \mathrm{~kg}$, pulse 59 beats per minute. He was then followed up with monthly calcium check, counselled to drink 3 to 41 of fluid per day. He was recommended to continue oral paracetamol use for symptom relief when needed and educated about symptoms of hypercalcemia to watch out for.

Two and a half years before this report date, while being evaluated for a globus sensation, it was noted that the patient's parathyroid hormone (PTH) level had risen over the previous two checks (Fig. 2) and was then $12.7 \mathrm{pmol} / 1$ (reference range for PTH: 1.6-6.9 pmol/1), his corrected ionized serum calcium level at $2.9 \mathrm{mmol} / \mathrm{l}$. His eGFR was 96 $\mathrm{ml} / \mathrm{min}$; vitamin D level was $52 \mathrm{nmol} / \mathrm{l}$ (optimal), his urinary calcium and creatinine clearance ratio was 0.0028 (reference range: $<0.01$ ) (Fig. 3), twenty-four-hour urinary calcium level was $1.9 \mathrm{mmol}$ (reference range $2.5-8.0 \mathrm{mmol} / 24 \mathrm{~h}$ ). A renal calculus measuring $5 \mathrm{~mm}$ was picked up on a routine renal ultra-sound scan, not causing an obstruction. A Sestamibi scan of the parathyroid glands showed two areas of hyperactivity on the right and one on the left. Two serial ultrasound studies of the thyroid glands done also noted a nodule within the right thyroid lobe adjacent to the thyroid isthmus that appeared slightly iso- to hyperechoic with predominately peripheral vascularity, it measured about $8.8 \times 6.2 \mathrm{~mm}$, an increase in size from $4.9 \times 3.6 \mathrm{~mm}$ in the previous examination. A well-defined $9.7 \times 6.8 \mathrm{~mm}$ hypoechoic lesion with suggestion of cribriform appearance within the inferolateral aspect of the left thyroid lobe with no detectable intra nodular vascularity was also noted and was considered a U2 nodule.

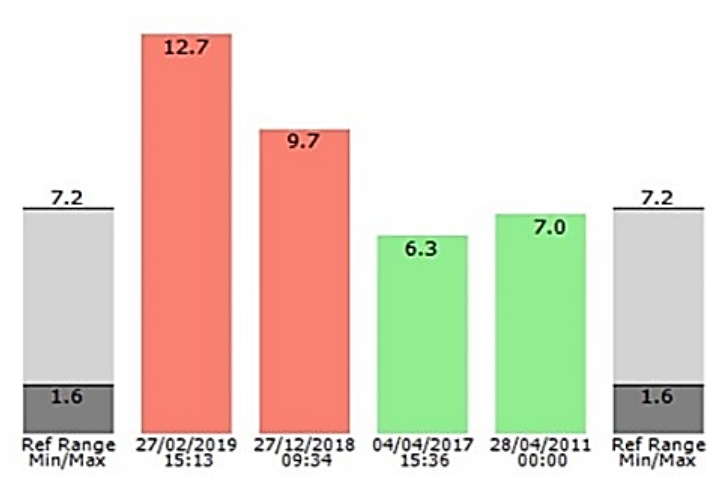

Fig. 2. Showing elevated PTH levels.

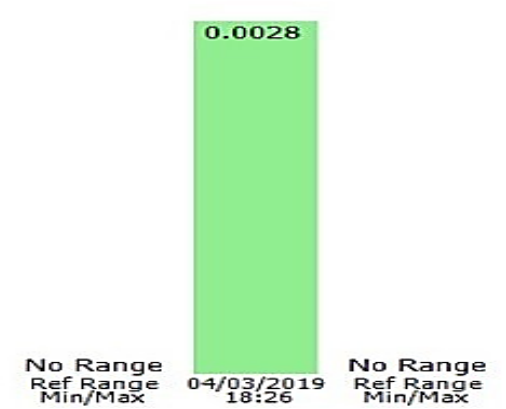

Fig. 3. Showing a reduced urine calcium:creatinine ratio (reference range $<0.01)$.

Based on the above lab results and scan report, a case of thyroid nodules and hyperparathyroidism complicating a preexisting provisional diagnosis of familial hypocalciuric hypercalcemia was made. The patient was commenced on oral cinacalcet as a medical treatment to lower the elevated parathyroid hormone level while surgical option for managing his parathyroid hypertrophy was being considered [2]. He was reviewed in an otorhinolaryngology clinic and a request was made for a genetic confirmation of $\mathrm{FHH}$ before considering surgical options. A result consistent with a diagnosis of Familial Hypocalciuric Hypercalcemia type 1 was received after being referred for the genetic sequencing study. He was heterozygous for the calcium sensing receptor (CASR) frameshift variant causing FHH (Fig. 4) [3]. He underwent a right subtotal thyroidectomy and right parathyroidectomy was done.

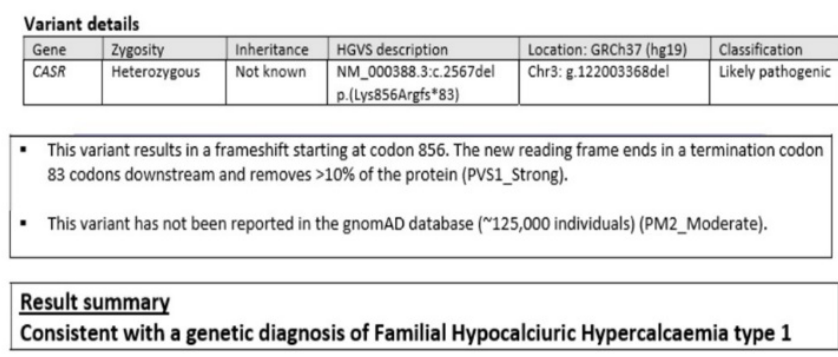

Fig. 4. Showing genetic sequencing report confirming FHH type 1.

In the immediate period post-surgery, cinacalcet was electively continued and parathyroid hormone levels and serum ionized calcium levels were noted within reference ranges. He was counselled to communicate the news of the diagnosis of FHH to his blood-line relatives and that they may need genetic screening also. Six months post-surgery, he was 
noted with a return of hypercalcemia (serum ionized calcium level of $2.8 \mathrm{mmol} / \mathrm{l}$ ) and hypophosphatemia (of $0.61 \mathrm{mmol} / \mathrm{l}$ ) while on treatment with oral cinacalcet. Cinacalcet use was subsequently withdrawn and a repeat check on serum parathyroid hormone level was noted to be $9.3 \mathrm{pmol} / 1$ (reference range 1.6-7.2 pmol/l). Patient was then re-referred to the otorhinolaryngology unit for further surgical review.

\section{DisCUSSION}

Familial hypocalciuric hypercalcemia is a rare clinical abnormality of impaired calcium homeostasis, with genetic importance. FHH Type 1 (the more common type) is associated with mutation of the extracellular calcium-sensing receptors in the parathyroid glands and kidneys. FHH raises the set point of serum calcium levels to higher-than-normal ranges. Patients are usually asymptomatic, and treatment is often not offered to normalize calcium levels. Symptomatic management is often offered depend on the patient's symptoms [4].

FHH Type 1 results from a loss-of-function mutation of the receptors that sense calcium ions (CASR) genes in renal tubules and on the parathyroid glands. FHH type 2 occurs because of mutation to Guanine Nucleotide-binding protein subunit Alpha-11 (GNA11) gene, while Type 3 occurs due to changes to Adaptor-Related Protein Complex 2 Sigma 1 (AP2S1) gene subunit [5]. The first FHH type described above was the type detected on genetic screening of this patient being reported.

Superimposed or co-existing FHH and hyperparathyroidism are even a rarer occurrence, and limited research into the association of these two clinical entities have been done. The biochemical picture in the evaluation of these two clinical entities occurring together is elevated serum calcium levels, hypophosphatemia, elevated parathormone levels and low urinary calcium levels. Lesley S.E et al. [6] in 2012 described two similar cases with FHH (one of whom had a genetically confirmed CASR mutation) and primary hyperparathyroidism. Their publication conveyed that surgical intervention helps to reduce calcium levels, improve symptoms, and prevent complications from the hypercalcemia but may not completely normalize serum calcium levels [6].

Some patients have been reported to have undergone parathyroid surgeries for identified parathyroid pathologies and hypercalcemia and found not to have settled calcium levels due to previously undiagnosed FHH. Such was the case in the report by Forde HE et al. in 2014 [7]. In our case, the intended surgery is post-dating the genetic confirmation of FHH. P. J. Robinson et al. [8] in 1990 emphasized the importance of delineating the possibility of an underlying FHH before undertaking parathyroid procedures for primary hyperparathyroidism. This was his submission after a failed neck exploration for presumed primary hyperparathyroidism. They went on to discover seven affected family members in the patient's kindred line who tested positive for FHH [8].

Rarely seen severe hyperparathyroidism due to homozygous mutation of FHH has been described in neonates. When this is the case, surgery (subtotal parathyroidectomy) is often offered to these newborns [9]. Other possible indications for surgery (subtotal parathyroidectomy) in $\mathrm{FHH}$ include relapsing pancreatitis and severe hypercalcemia values $(>3.5 \mathrm{mmol} / \mathrm{l})[10]$.

Establishing a link between the development of parathyroid hyperplasia or adenoma on a background of familial hypocalciuric hypercalcemia appears to be getting an increased reporting and remains to be scientifically explained. If there is a plausible scientific explanation for this, then patients with FHH will need counselling that this is a possibility, and that surgery may be anticipated if this occurs [11]. Evidence for this association will need to be demonstrated beyond the pages of case reports or case series to higher scientific or evidence-based studies.

\section{CONCLUSION AND LEARNING PoInTS}

1. While evaluating hypercalcemia patients, a rare situation of FHH should be borne in mind. Hence, urinary calcium studies should be included as part of initial workup tests for hypercalcemia evaluation.

2. An onward development of hyperparathyroidism complicating $\mathrm{FHH}$ in patients with this condition should be borne in mind due to increasing evidence about this association.

3. Medical treatment or surgical intervention may become added modalities for a condition that is largely managed conservatively if $\mathrm{FHH}$ becomes complicated by hyperparathyroidism.

4. Evaluation and genetic screening should be offered to blood-line relatives of patients who are diagnosed with FHH.

\section{ACKNOWLEDGEMENT}

The authors acknowledge the kind gesture of our patient for consenting to have this case shared to increase the body of knowledge in the medical field.

\section{REFERENCES}

[1] A. Alkundi, R. Momoh, "Hyperparathyroidism complicating a preexisting diagnosis of Familial Hypocalciuric Hypercalcemia (FHH)," https://www.endocrine-abstracts.org/ea/0073/ea0073ep33.

[2] H. J. Timmers, M. Karperien, M. A. T. Hamdy, H. De Boer, A. R. Hermus, "Normalization of serum calcium by cinacalcet in a patient with hypercalcaemia due to a de novo inactivating mutation of the calcium-sensing receptor," https://doi.org/10.1111/j.13652796.2006.01684.x.

[3] P. H. Pissen, S. E. Christensen, L. Heickendorff, K. Brixen, L. Mosekeide, "Molecular Genetic Analysis of the Calcium Sensing Receptor Gene in Patients Clinically Suspected to Have Familial Hypocalciuric Hypercalcemia: Phenotypic Variation and Mutation Spectrum in a Danish Population," https://doi.org/10.1210/jc.20070322 .

[4] J. Y. Lee, D. M. Shoback, "Familial hypocalciuric hypercalcemia and related disorders," Best Pract Res Clin Endocrinol Metab., 32(5):609619, 2018. doi:10.1016/j.beem.2018.05.004.

[5] M. Aashiq, A. J. Malallah, F. Khan, M. Alsada, "Clinical and Biochemical Features in a Case of Familial Hypocalciuric Hypercalcemia Type 3 with AP2S1 Gene Mutation in Codon Arg15His," https://www.hindawi.com/journals/cripe/2020/7312894/.

[6] L. S. Eldeiry, D. T. Ruan, E. M. Brown, J. M. Gaglia, J. R. Garber, "Primary hyperparathyroidism and familial hypocalciuric hypercalcemia: relationships and clinical implications," Endocr Pract, 18(3):412-417, 2012. doi:10.4158/EP11272.RA.

[7] H. E. Forde, A. D. Hill, D. Smith, "Parathyroid adenoma in a patient with familial hypocalciuric hypercalcaemia," BMJ Case Rep, 2014bcr2014206473, 2014. doi:10.1136/bcr-2014-206473. 
[8] P. J. Robinson, R. J. Corall, "The importance of distinguishing familial hypocalciuric hypercalcaemia from asymptomatic primary hyperparathyroidism prior to neck exploration," https://onlinelibrary.wiley.com/doi/abs/10.1111/j.1365-

2273.1990.tb00447.x.

[9] M. Blackburn. "Primary hyperparathyroidism and familial hyperparathyroid syndromes".

https://www.racgp.org.au/afpbackissues/2007/200712/200712Blackbu rn.pdf.

[10] E. Shane, D. Irani, "Hypercalcemia: Pathogenesis, Clinical Manifestations, Differential Diagnosis, and Management," https://www.homepages.ucl.ac.uk/ ucgatma/Anat3048/PAPERS\%20e tc/ASBMR\%20Primer\%20Ed\%206/Ch\%2026-41\%20\%20Disorders\%20of\%20Serum\%20Minerals.pdf/

[11] M. Papadakis, N. Meurer, T. Margariti, A. Meyer, N. Weyerbrock, C. Dotzenrath, "A novel mutation of the calcium-sensing receptor gene in a German subject with familial hypocalciuric hypercalcemia and primary hyperparathyroidism".

https://link.springer.com/content/pdf/10.14310/horm.2002.1711.pdf. 